\title{
MIMO Broadcast Channel with Arbitrarily Varying Eavesdropper Channel: Secrecy Degrees of Freedom
}

\author{
Xiang $\mathrm{He}^{*}$, Ashish Khisti ${ }^{\dagger}$, Aylin Yener* \\ * Electrical Engineering Department, The Pennsylvania State University, University Park, PA 16802 \\ ${ }^{\dagger}$ Dept. of Electrical and Computer Engineering, University of Toronto, Toronto, ON, M5S 3G4, Canada \\ hexiang@ieee.org, akhisti@comm.utoronto.ca,yener@psu.edu
}

\begin{abstract}
A two-receiver MIMO broadcast-wiretap channel is considered where the channel state of the eavesdropper is arbitrarily varying. It is assumed that the eavesdropper knows this channel state perfectly whereas the legitimate nodes have no knowledge of it. It is further assumed that the eavesdropper experiences no additive noise. The channel between the transmitter and the two legitimate receivers is a constant MIMO Gaussian broadcast channel. This paper establishes the secrecy degrees of freedom region for transmitting a common-confidential message as well as a private-confidential message to each receiver. It is observed that a straightforward extension of single user random binning does not achieve the optimal secrecy degrees of freedom (s.d.o.f.) region. The proposed coding scheme that achieves the s.d.o.f. region involves simultaneous diagonalization of the channel matrices of the two legitimate receivers using the generalized singular value decomposition (GSVD) as well as a particular structured binning across codebooks that minimizes the rate of the fictitious message. While the focus is on achieving weak secrecy for ease of exposition, an outline is provided on how the results can be extended for achieving strong secrecy.
\end{abstract}

\section{INTRODUCTION}

All secrecy schemes are based on a small set of reasonable assumptions. The approach of studying secrecy problems using information theory was pioneered by Shannon in [1] and was later extended to different network models, see for example, [2]-[6]. The distinctive feature of this approach is that instead of assuming the adversary is computationally limited as in the case of computational security, secrecy is achieved relying solely on assumptions on the communication network, usually described in terms of network topology, channel states or the signal to noise ratio, allowing the adversary to be computationally unlimited. Such an approach therefore establishes the fundamental limits for secure communication rates, and identifies properties inherent to the communication network that can be leveraged to achieve positive secrecy rates for legitimate communication parties.

The possibility of achieving secure communication using multiple antennas has been studied extensively in literature. Most works assume (partial) knowledge of the eavesdropper channel state information and characterize the rates at which secure communication can take place, see [7]-[10] for example.

Since the eavesdropper does not transmit and hence its channel states are hard to obtain for legitimate communication parties, recent works [11], [12] have started to consider the case where the eavesdropper channel is arbitrarily varying and its channel states are known to the eavesdropper only. Reference [12] has studied the single-user Gaussian MIMO wiretap channel and found its secrecy degrees of freedom, which is a high SNR characterization of the capacity of this model. Reference [12] has also provided the secrecy degrees of freedom region for a two-receiver Gaussian MIMO broadcast channel where each legitimate node has the same number of antennas, which is obtained as a straightforward extension of the single user case. In both cases, only the number of antennas employed by the eavesdropper is known to the transmitter. This assumption can be justified for the scenarios where the eavesdropping device is small and hence is unlikely to employ more than a certain number of antennas.

In this work, we consider the general setting where the nodes have any number of antennas and characterize the secrecy degrees of freedom region for the two-receiver Gaussian MIMO broadcast channel. The achievability proof is not a straightforward extension of [12] which involves constructing a vector codebook sampled in an i.i.d. fashion and random binning. A direct construction of two codebooks in this manner introduces an independent randomization for each codebook and creates higher than necessary interference between the legitimate users. Instead, our approach involves carefully transmitting a fictitious message, of just enough rate, in a common subspace between the two users so that it can be simultaneously useful for providing secrecy for both users. This scheme can be viewed as inducing a structured binning of the codebooks to minimize the size of each bin.

\section{SYSTEM MODEL}

We consider a MIMO Broadcast (BC) wiretap channel with two receivers, as shown in Figure 1. We assume that the transmitter has $N_{T}$ antennas. For $t=1,2$, receiver $t$ has $N_{R_{t}}$ antennas, The eavesdropper has $N_{E}$ antennas. During the $i$ th channel use, the channel is:

$$
\begin{aligned}
& \mathbf{Y}_{t}(i)=\mathbf{H}_{t} \mathbf{X}(i)+\mathbf{Z}_{t}(i), t=1,2 \\
& \tilde{\mathbf{Y}}(i)=\tilde{\mathbf{H}}(i) \mathbf{X}(i)
\end{aligned}
$$

where $\mathbf{Y}_{t}(i), t=1,2$ denote the signals received at the legitimate receivers, and $\tilde{\mathbf{Y}}(i)$ denotes the received signal at the eavesdropper. $\mathbf{H}_{t}, t=1,2$ and $\tilde{\mathbf{H}}(i)$ are the channel 
matrices. $\mathbf{Z}_{t}, t=1,2$ is the additive Gaussian noise observed by the intended receiver $t$, which is composed of independent rotationally invariant complex Gaussian random variables with unit variance. $\tilde{\mathbf{H}}(i)$ is unknown to the legitimate parties. $\mathbf{H}_{t}, t=1,2$ are known by both the legitimate parties and the eavesdropper(s). ${ }^{1}$

For clarity, we shall use $\gamma$ to represent a sequence of $\{\tilde{\mathbf{H}}(i)\}$ and use $\left\{\tilde{\mathbf{Y}}_{\gamma}(i)\right\}$ to represent the outputs of the eavesdropper channel that corresponds to this sequence of eavesdropper channel states.

Each receiver $t$ receives a confidential message $W_{t}$, and a common confidential message $W_{0}$ from the transmitter over $\bar{n}$ channel uses. $W_{0}, W_{1}, W_{2}$ must be kept confidential from the eavesdropper. Let $\mathcal{W}_{i}, i=0,1,2$ denote the alphabet for $W_{i}$. $\left|\mathcal{W}_{i}\right|$ denotes the cardinality of $\mathcal{W}_{i}$.

The average power constraint for the transmitter is

$$
\lim _{\bar{n} \rightarrow \infty} \frac{1}{\bar{n}} \sum_{i=1}^{\bar{n}} \operatorname{trace}\left(\mathbf{X}(i)(\mathbf{X}(i))^{H}\right) \leq \bar{P}
$$

We assume the eavesdropper channel state information sequence $\{\tilde{\mathbf{H}}(i)\}$ is independent from $\mathbf{X}$. In this case, as shown in [12], the secrecy constraint can be defined as:

$$
\lim _{\bar{n} \rightarrow \infty} I\left(W_{0}, W_{1}, W_{2} ; \tilde{\mathbf{Y}}_{\gamma}^{\bar{n}}\right)=0, \quad \forall \gamma
$$

where $\gamma$ is used to index the eavesdropper channel state sequence. We require the limit in (4) to be uniform over all possible sequences of eavesdropper channel states [12].

The secrecy rate for the message $W_{i}, R_{s, i}$, is defined as $R_{s, i}=\lim _{\bar{n} \rightarrow \infty} \frac{1}{\bar{n}} H\left(W_{i}\right), i=0,1,2$ such that $\left\{W_{0}, W_{t}\right\}$ can be reliably decoded by receiver $t, t=1,2$.

In this paper, we use the secrecy degrees of freedom (s.d.o.f.) region as a characterization of the high SNR behavior of the secrecy capacity for this channel. The s.d.o.f. region is defined as:

$$
\left\{\left(d_{0}, d_{1}, d_{2}\right): d_{i}=\limsup _{\bar{P} \rightarrow \infty} \frac{R_{s, i}}{\log _{2} \bar{P}}, i=0,1,2\right\}
$$

\section{MAIn RESUlT}

Theorem 1: Let $r_{1}, r_{2}$ be the rank of $\mathbf{H}_{1}$ and $\mathbf{H}_{2}$ respectively. Let $r_{0}$ be the rank of $\left[\mathbf{H}_{1}^{T}, \mathbf{H}_{2}^{T}\right]^{T}$. The secrecy degrees of freedom region for the MIMO broadcast wiretap channel in Figure 1 is given by

$$
\begin{aligned}
& 0 \leq d_{j}, \quad j=0,1,2 \\
& 0 \leq d_{0}+d_{i} \leq \max \left\{0, r_{i}-N_{E}\right\}, i=1,2 \\
& 0 \leq d_{0}+d_{1}+d_{2} \leq \max \left\{0, r_{0}-N_{E}\right\}
\end{aligned}
$$

Remark 1: The result here can be viewed as a Gaussian model counterpart of [13] that establishes the secrecy degrees of freedom for a class of deterministic memoryless broadcast channels. However, the result in [13] is based on the use

\footnotetext{
${ }^{1}$ Since the eavesdropper channel is arbitrarily varying, the model includes the case of having any number of non-colluding eavesdroppers.
}

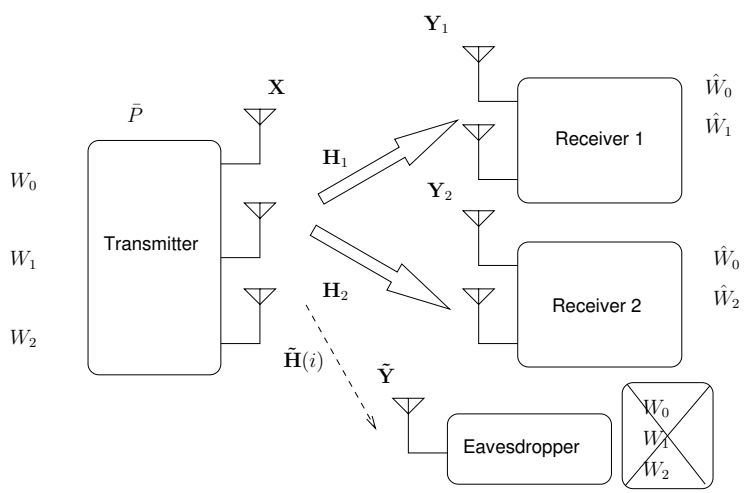

Fig. 1. The MIMO Broadcast Wiretap Channel where $N_{T}=3, N_{R_{1}}=$ $N_{R_{2}}=2, N_{E}=1$.

of rank metric codes and does not generalize to Gaussian channels. We also observe that when $N_{E}=0$, i.e., there is no eavesdropper, the result here can be shown to be equivalent to the rate region derived in [14] by applying Fourier-Motzkin elimination on $[14,(50)-(53)]$, the constraints on $\eta, \delta$ below [14, (50)-(53)], and $d_{i} \geq 0, i=0,1,2$.

\section{Motivating Example: $3 \times 2 \times 2 \times 1$ Channel}

Consider the example in Figure 1 where $N_{t}=2, N_{E}=1$ and $N_{R_{1}}=N_{R_{2}}=2$. Assume that $r_{1}=r_{2}=2$ and $r_{0}=3$. As we discuss in the sequel, after an appropriate transformation, the channel matrices of the two legitimate receivers reduce to:

$$
\mathbf{H}_{1}=\left[\mathbf{I}_{(2 \times 2)}, \mathbf{0}_{(2 \times 1)}\right], \quad \mathbf{H}_{2}=\left[\mathbf{0}_{(2 \times 1)}, \mathbf{I}_{(2 \times 2)}\right]
$$

while the effective channel matrix of the eavesdropper is an arbitrary rank one matrix. Reference [12] shows that there exists a codebook $\mathcal{C}_{1}$ that can be transmitted over the first and the second antenna to achieve $d_{1}=1$, and there exists a codebook $\mathcal{C}_{2}$ that can be transmitted over the second and the third antenna to achieve $d_{2}=1$. However, since $W_{1}$ and $W_{2}$ are independent, the signals that $\mathcal{C}_{1}$ uses to represent $W_{1}$ over the second antenna in general do not agree with the signals that $\mathcal{C}_{2}$ uses to represent $W_{2}$ over this antenna, causing a conflict. Thus, we need to construct a new scheme.

Our proposed scheme resolves this conflict by constructing three codebooks, one for each link. A codebook on the second link $\mathcal{C}_{E}$ is used to transmit a fictitious message $W_{E}$ via a codeword $X_{E}^{n}\left(W_{E}\right)$. An independent codebook on the first link $\mathcal{C}_{1}$, of twice the rate, is used to transmit a codeword $X_{1}^{n}\left(W_{E}, W_{1}\right)$ while another codebook $\mathcal{C}_{2}$ on the third link is used to transmit a codeword $X_{2}^{n}\left(W_{E}, W_{2}\right)$. It can be verified that both users 1 and 2 can decode $\left(W_{1}, W_{E}\right)$ and $\left(W_{2}, W_{E}\right)$ respectively whereas the secrecy analysis reveals that both $\left(W_{1}, W_{2}\right)$ are protected from the eavesdropper. In the next section we generalize this scheme to arbitrary number of antennas and a common message $W_{0}$.

Note that the proposed construction has three independent messages. In contrast, the naive extension of singleuser random binning consists of four independent mes- 


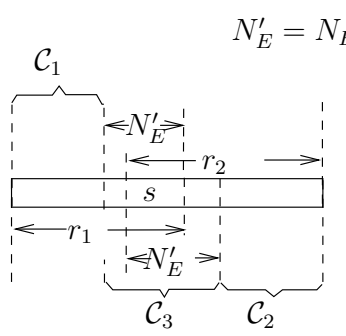

(a)

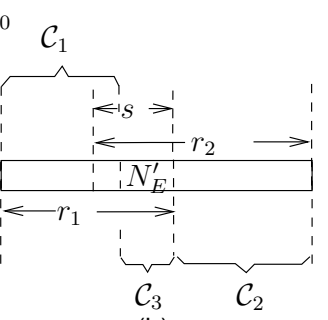

(b)
Fig. 2. Codebook generation: (a) $s \leq N_{E}^{\prime} \leq \min \left\{r_{1}, r_{2}\right\}$ (b) $0 \leq N_{E}^{\prime}<s$. $s=r_{1}+r_{2}-r_{0}$.

sages: one message for each user and one message from random codeword selection in each bin and forces the users to decode more information than is necessary. Our construction induces structured binning across the codebooks: given a choice of messages $w_{0}, w_{1}, w_{2}$ the bin index $B_{w_{0}, w_{1}, w_{2}}$ consists of all sequences of the form $\left\{\bigcup_{w_{E}}\left(x_{1}^{n}\left(w_{1}, w_{0}, w_{E}\right), x_{E}^{n}\left(w_{0}, w_{E}\right), x_{2}^{n}\left(w_{2}, w_{0}, w_{E}\right)\right)\right\}$. The following lemma, whose proof will be omitted due to space constraints is used in the secrecy analysis.

Lemma 1: Let $\left|\mathcal{W}_{E}\right|$ denote the cardinality of the set of possible values for the fictitious message $W_{E}$. Then

1) The size of each $B_{w_{0}, w_{1}, w_{2}}$ equals $\left|\mathcal{W}_{E}\right|$.

2) The codewords within $B_{w_{0}, w_{1}, w_{2}}$ are i.i.d..

\section{Proof Outline}

The converse follows from standard pairwise upper bound considerations, see [13]. We focus on the achievability proof here.

Define $d(x)$ as $d(x)=\limsup _{\bar{P} \rightarrow \infty} \frac{x}{\log _{2} \bar{P}}$. For ease of explanation, we shall first prove Theorem 1 in terms of the following secrecy requirement:

$$
\lim _{\bar{n} \rightarrow \infty} \frac{1}{\bar{n}} d\left(I\left(W_{0}, W_{1}, W_{2} ; \tilde{\mathbf{Y}}_{\gamma}^{\bar{n}}\right)\right)=0, \quad \forall \gamma,
$$

and restrict ourselves to the case where the eavesdropper channel state is arbitrary but does not change over time. Later, in Section V-F, we shall outline the techniques required to strengthen the result for the strong secrecy case (4) when the eavesdropper channel is arbitrarily varying.

In the proof, we focus on a special form of channel model. It can be shown through generalized singular value decomposition [8], [14] that the general channel model can be converted to the form we are considering while preserving the degrees of freedom region. In this special form, $N_{T}=r_{0}$ and $\overline{\mathbf{\Sigma}}_{t}$ is a $N_{R_{t}} \times r_{0}$ matrix:

$$
\begin{aligned}
& \mathbf{Y}_{t}(i)=\overline{\boldsymbol{\Sigma}}_{t} \mathbf{X}_{\left(r_{0} \times 1\right)}(i)+\mathbf{Z}_{t}(i), t=1,2 \\
& \tilde{\mathbf{Y}}(i)=\tilde{\mathbf{H}}_{\left(N_{E} \times r_{0}\right)}(i) \mathbf{X}_{\left(r_{0} \times 1\right)}(i)
\end{aligned}
$$

The only nonzero elements in $\overline{\boldsymbol{\Sigma}}_{t}$ are the first $r_{1}$ leading elements on the main diagonal line of $\overline{\boldsymbol{\Sigma}}_{1}$ and the last $r_{2}$ elements on the main diagonal line of $\overline{\boldsymbol{\Sigma}}_{2}$. These nonzero elements are all positive and share the same value, which we denote with $\tau_{\min }^{2}$. Since no user can achieve a positive secrecy rate when $r_{0} \leq N_{E}$, we assume $r_{0}>N_{E}$ and consider $\tilde{\mathbf{H}}$ that has the following form:

$$
\tilde{\mathbf{H}}=\left[\mathbf{I}_{N_{E} \times N_{E}}, \mathbf{0}_{N_{E} \times\left(r_{0}-N_{E}\right)}\right] \mathbf{U}_{E}(i)
$$

where $\mathbf{U}_{E}(i)$ is a unitary matrix only known to the eavesdropper. As in [12], [15], we then introduce artificial noise into $\mathbf{X}$ in (11) and (12) by computing $\mathbf{X}$ as:

$$
\mathbf{X}(i)=\tilde{\mathbf{X}}_{\left(r_{0} \times 1\right)}(i)+\mathbf{N}(i)
$$

where $\mathbf{N}$ is the $r_{0} \times 1$ artificial noise vector consisting of independent rotationally invariant complex Gaussian random variables with zero mean and unit variance. The codebook is designed to transmit $\tilde{\mathbf{X}}$.

Define $N_{E}^{\prime}$ as $N_{E}^{\prime}=N_{E}+d_{0} . s=r_{1}+r_{2}-r_{0}$. As in [13], in the proof we consider two cases:

1) $s \leq N_{E}^{\prime} \leq \min \left\{r_{1}, r_{2}\right\}$. In this case (8) is not active and to prove the region is achievable we only need to show for a given value of $d_{0}$, the pair $\left(d_{1}=r_{1}-N_{E}^{\prime}, d_{2}=\right.$ $\left.r_{2}-N_{E}^{\prime}\right)$ is achievable.

2) $0<N_{E}^{\prime}<s$. In this case (8) is active. To prove the region is achievable we only need to show for a given value of $d_{0}$ the two corner points $\left(d_{1}=r_{1}-N_{E}^{\prime}, d_{2}=\right.$ $\left.\tilde{r}_{2}\right)$ and $\left(d_{1}=\tilde{r}_{1}, d_{2}=r_{2}-N_{E}^{\prime}\right)$ are achievable. We shall prove the pair $\left(d_{1}=r_{1}-N_{E}^{\prime}, d_{2}=\tilde{r}_{2}\right)$ is achievable since the proof for the other pair is similar.

\section{A. Input Distribution}

Let $C(x)=\log _{2}(1+x)$. Define $P$ such that $P+r_{0}$ is proportional to $\bar{P}$. Define $R$ as $R=C\left(\tau_{\min }^{2}\left(P / r_{0}\right) /\left(\tau_{\text {min }}^{2}+1\right)\right)$. We shall allocate a total power of $r_{0}$ units on artificial noise $\mathbf{N}$ in (14) and $P / r_{0}$ units on each antenna for $\tilde{\mathbf{X}}$.

As mentioned in Section IV, we shall divide the antennas into different groups, which will be described in Section V-B, and generate codebooks for each group. The input distribution we use to generate codebooks is a truncated Gaussian distribution: For a group that contains $k$ antennas, let $\tilde{\mathbf{X}}_{[k]}$ denote a random vector formed by any $k$ components of $\tilde{\mathbf{X}}$ in (14). For a positive constant $\varepsilon_{P}$, define $Q_{\tilde{\mathbf{X}}_{[k]}}(\mathbf{x})$ be a $k$-dimensional rotationally invariant complex Gaussian distribution with covariance matrix $\left(P\left(1-\varepsilon_{P}\right) / r_{0}\right) \mathbf{I}_{(k \times k)}$. We define the following truncated $n$-letter input distribution $Q_{\tilde{\mathbf{X}}_{[k]}^{n}}\left(\mathbf{x}^{n}\right)$ used to generate the codebooks: Let $\mathbf{x}_{i}$ denote the $i$ th component of $\mathbf{x}^{n} \cdot Q_{\tilde{\mathbf{X}}_{[k]}^{n}}\left(\mathbf{x}^{n}\right)$ is given by:

$$
Q_{\tilde{\mathbf{X}}_{[k]}^{n}}\left(\mathbf{x}^{n}\right)=\mu_{n, k, \varepsilon_{P}}^{-1} \varphi\left(\mathbf{x}^{n}\right) \prod_{i=1}^{n} Q_{\tilde{\mathbf{X}}_{[k]}}\left(\mathbf{x}_{i}\right)
$$

where $\mu_{n, k, \varepsilon_{P}}=\int \varphi\left(\mathbf{x}^{n}\right) \prod_{i=1}^{n} Q_{\tilde{\mathbf{x}}_{[k]}}\left(\mathbf{x}_{i}\right) d \mathbf{x}^{n}$ and $\varphi\left(\mathbf{x}^{n}\right)$ equals 1 if $\frac{1}{n}\left\|\mathbf{x}^{n}\right\|^{2} \leq k P / r_{0}$ and equals 0 otherwise.

\section{B. Codebook Generation}

Let $\left\{\delta_{n}\right\}$ be a positive sequence of $n$ that can be made arbitrarily small. Define $s=r_{1}+r_{2}-r_{0} . \tilde{r}_{t}=r_{t}-s, t=1,2$. The codebook generation depends on the values of $N_{E}^{\prime}$ and is illustrated in Figure 2. It is understood that when $k$ in (15) is zero, the codebook is empty. 
1) $s \leq N_{E}^{\prime} \leq \min \left\{r_{1}, r_{2}\right\}$.

a) For $t=1,2, \mathcal{C}_{t}$ is composed of $2^{n\left(r_{t} R-2 \delta_{n}\right)}$ i.i.d.

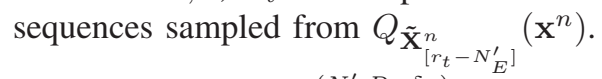

b) $\mathcal{C}_{3}$ is composed of $2^{n\left(N_{E}^{\prime} R-\delta_{n}\right)}$ i.i.d. sequences sampled from $Q_{\tilde{\mathbf{X}}_{\left[2 N_{E}^{\prime}-s\right]}^{n}}\left(\mathbf{x}^{n}\right)$.

Each codeword in $\mathcal{C}_{3}$ is labeled with $i_{3}$ and $j_{3} .0 \leq i_{3} \leq$ $2^{n\left(N_{E} R-\delta_{n}\right)}-1.0 \leq j_{3} \leq 2^{n\left(d_{0} R\right)}-1 . i_{3}$ shall play the role of $W_{E}$ in Section IV.

For $t=1,2$, each codeword in $\mathcal{C}_{t}$ is labeled with $i_{t}$ and $j_{t}$, $0 \leq i_{t} \leq 2^{n\left(N_{E}^{\prime} R-\delta_{n}\right)}-1,0 \leq j_{t} \leq 2^{n\left(\left(r_{t}-N_{E}^{\prime}\right) R-\delta_{n}\right)}-1$.

2) $0 \leq N_{E}^{\prime}<s$ : To prove the achievability of the corner point $\left(d_{1}=r_{1}-N_{E}^{\prime}, d_{2}=\tilde{r}_{2}\right), \mathcal{C}_{t}, 1 \leq t \leq 3$ are generated as follows:

a) $\mathcal{C}_{1}$ is composed of $2^{n\left(r_{1} R-2 \delta_{n}\right)}$ i.i.d. sequences sampled from $Q_{\tilde{\mathbf{X}}_{\left[r_{1}-N_{E}^{\prime}\right]}^{n}}\left(\mathbf{x}^{n}\right)$.

b) $\mathcal{C}_{2}$ is composed of $2^{n\left(\left(\tilde{r}_{2}+N_{E}^{\prime}\right) R-2 \delta_{n}\right)}$ i.i.d. sequences sampled from $Q_{\tilde{\mathbf{X}}_{\left[\tilde{r}_{2}\right]}^{n}}\left(\mathbf{x}^{n}\right)$.

c) $\mathcal{C}_{3}$ is composed of $2^{n\left(N_{E}^{\prime} R-\delta_{n}\right)}$ i.i.d. sequences sampled from $Q_{\tilde{\mathbf{X}}_{\left[N_{E}^{\prime}\right]}^{n}}\left(\mathbf{x}^{n}\right)$.

We then label $\mathcal{C}_{3}$ with $\left(i_{3}, j_{3}\right)$ as described in the previous sub-section, Section V-B1. $\mathcal{C}_{1}$ is labeled with $\left(i_{1}, j_{1}\right)$ as described in Section V-B1. Each codeword in $\mathcal{C}_{2}$ is labeled with $i_{2}$ and $j_{2}: 0 \leq i_{2} \leq 2^{n\left(N_{E}^{\prime} R-\delta_{n}\right)}-1,0 \leq j_{2} \leq 2^{n\left(\tilde{r}_{2} R-\delta_{n}\right)}-1$.

\section{Encoder}

Since $\left\{i_{3}, j_{3}\right\}$ has the same cardinality as $\left\{i_{t}\right\}$, we can define one-to-one mapping between these two. Denote the mapping with $h_{t}$.

a) The encoder chooses $i_{3}$ based on uniform distribution.

b) The encoder chooses $j_{3}=W_{0}$.

c) For $t=1,2$, we compute $\left\{i_{t}, j_{t}\right\}$ as follows:

$$
i_{t}=h_{t}\left(i_{3}, j_{3}\right), \quad j_{t}=W_{t}
$$

1) $s \leq N_{E}^{\prime} \leq \min \left\{r_{1}, r_{2}\right\}$ : The codeword with label $i_{1}, j_{1}$ is chosen from $\mathcal{C}_{1}$ and transmitted over the first $r_{1}-N_{E}$ components of $\tilde{\mathbf{X}}$ in (14).

The codeword with label $i_{2}, j_{2}$ is chosen from $\mathcal{C}_{2}$ and transmitted over the last $r_{2}-N_{E}$ component of $\tilde{\mathbf{X}}$ in (14).

The codeword with label $i_{3}$ is chosen from $\mathcal{C}_{3}$ and transmitted over the remaining $2 N_{E}-s$ components of $\tilde{\mathbf{X}}$ in (14).

2) $0 \leq N_{E}^{\prime}<s$ : As in Section V-C1, the codeword with label $i_{1}, j_{1}$ is chosen from $\mathcal{C}_{1}$ and transmitted over the first $r_{1}-N_{E}$ component of $\tilde{\mathbf{X}}$ in (14).

The codeword with label $i_{2}, j_{2}$ is chosen from $\mathcal{C}_{2}$ and transmitted over the last $\tilde{r}_{2}$ component of $\tilde{\mathbf{X}}$ in (14).

The codeword with label $i_{3}$ is chosen from $\mathcal{C}_{3}$ and transmitted over the remaining $N_{E}$ components of $\tilde{\mathbf{X}}$ in (14).

\section{Decoder}

1) $s \leq N_{E}^{\prime} \leq \min \left\{r_{1}, r_{2}\right\}$ : For $t=1,2$,

a) Receiver $t$ first decodes the codeword from $\mathcal{C}_{3}$.

In this step, for receiver 1 , the decoder takes the last $N_{E}^{\prime}$ components of $\mathbf{Y}_{t}$ in (11) as inputs. For receiver 2, the decoder takes the first $N_{E}^{\prime}$ components of $\mathbf{Y}_{t}$ in (11) as inputs.

Receiver $t$ then uses the label of the decoded codeword as its estimate for $i_{3}, j_{3}$, which is denoted by $\hat{i}_{3, t}, \hat{j}_{3, t}$. The estimate for the common confidential message $W_{0}$, denoted by $\hat{W}_{0, t}$, is then given by $\hat{j}_{3, t}$. The estimate for the label $i_{t}$, denoted by $\hat{i}_{t}$, is then given by $h_{t}\left(\hat{i}_{3, t}, \hat{j}_{3, t}\right)$.

b) Receiver $t$ then estimates the transmitted codeword from $\mathcal{C}_{t}$ based on the remaining $r_{t}-N_{E}^{\prime}$ components of $\mathbf{Y}_{t}$ in (14). Note that only those codewords in $\mathcal{C}_{t}$ whose label $i_{t}=\hat{i}_{t}$ need to be considered. From the labels of the most likely codeword in $\mathcal{C}_{t}$, receiver $t$ computes its estimate for label $j_{t}$, denoted by $\hat{j}_{t}$. Its estimate for message $W_{t}$, denoted by $\hat{W}_{t}$, is then given by $\hat{j}_{t}$.

2) $0 \leq N_{E}^{\prime}<s$ : Each receiver first computes $\hat{i}_{3, t}, \hat{j}_{3, t}$ as described in the previous subsection, Section V-D1. Receiver 1 computes $\hat{W}_{1}$ as in Section V-D1. Receiver 2 computes $\hat{W}_{2}$ as in Section V-D1 except that in step b), $r_{2}-N_{E}^{\prime}$ should be replaced by $\tilde{r}_{2}$.

\section{E. Secrecy Analysis}

In this section, we prove (10). Let \|\| denote the Euclidean distance. As in [12], define the following fictitious decoder:

$$
\phi_{\gamma, w_{0}, w_{1}, w_{2}}\left(\tilde{\mathbf{y}}^{n}\right)=\arg \max _{\mathbf{x}^{n} \in B_{w_{0}, w_{1}, w_{2}}}\left\|\tilde{\mathbf{y}}^{n}-\tilde{\mathbf{H}} \mathbf{x}^{n}\right\|
$$

which is the maximum likelihood decoder the eavesdropper can use to decode the transmitted signals when it assumes the secret message values are $W_{i}=w_{i}, i=0,1,2$.

Define $\eta_{\mathcal{C}, \gamma, w_{0}, w_{1}, w_{2}}$ as the probability of decoding error for this fictitious decoder, which is given by:

$$
\operatorname{Pr}\left(\phi_{\gamma, w_{0}, w_{1}, w_{2}}\left(\tilde{\mathbf{Y}}_{\gamma}^{n}\right) \neq \tilde{\mathbf{X}}^{n} \mid W_{i}=w_{i}, i=0,1,2\right)
$$

Define $\eta_{\mathcal{C}, \gamma}$ as the value of $\eta_{\mathcal{C}, \gamma, w_{0}, w_{1}, w_{2}}$ averaged over $w_{0}, w_{1}, w_{2}$, which is given by:

$$
\frac{1}{\left|\mathcal{W}_{0}\right| \times\left|\mathcal{W}_{1}\right| \times\left|\mathcal{W}_{2}\right|} \sum_{w_{i} \in \mathcal{W}_{i}, i=0,1,2} \eta_{\mathcal{C}, \gamma, w_{0}, w_{1}, w_{2}}
$$

Following [12], using Lemma 1, we have the following lemma. Its proof will be provided in the journal version of this work.

Lemma 2: There exists a codebook $\mathcal{C}$, such that $\lim _{n \rightarrow \infty} \eta_{\mathcal{C}, \gamma}=0$ uniformly over all $\gamma$.

For this codebook and for any $\gamma$, we have:

$$
\begin{aligned}
& H\left(W_{0}, W_{1}, W_{2} \mid \tilde{\mathbf{Y}}_{\gamma}^{n}\right)=I\left(W_{0}, W_{1}, W_{2} ; \tilde{\mathbf{X}}^{n} \mid \tilde{\mathbf{Y}}_{\gamma}^{n}\right) \\
= & H\left(\tilde{\mathbf{X}}^{n} \mid \tilde{\mathbf{Y}}_{\gamma}^{n}\right)-H\left(\tilde{\mathbf{X}}^{n} \mid \tilde{\mathbf{Y}}^{n}, W_{0}, W_{1}, W_{2}\right)
\end{aligned}
$$

(21) is lower bounded through Fano's inequality by

$$
H\left(\tilde{\mathbf{X}}^{n}\right)-I\left(\tilde{\mathbf{X}}^{n} ; \tilde{\mathbf{Y}}_{\gamma}^{n}\right)-1-\eta_{\mathcal{C}, \gamma} \log _{2}\left|\left\{\tilde{\mathbf{X}}^{n}\right\}\right|
$$

Due to Lemma 2 and the fact that $\log _{2}\left|\left\{\tilde{\mathbf{X}}^{n}\right\}\right|$ grows linearly with respect to $n$, we have

$$
\lim _{n \rightarrow \infty} \frac{1}{n} d\left(1+\eta_{\mathcal{C}, \gamma} \log _{2}\left|\left\{\tilde{\mathbf{X}}^{n}\right\}\right|\right)=0
$$


On the other hand, as shown in [12], we have:

$$
\lim _{n \rightarrow \infty} \frac{1}{n} d\left(I\left(\tilde{\mathbf{X}}^{n} ; \tilde{\mathbf{Y}}_{\gamma}^{n}\right)\right) \leq N_{E}
$$

For the first term in (22), we have:

$$
H\left(\tilde{\mathbf{X}}^{n}\right)=\log _{2}\left|\left\{i_{3}\right\}\right|+\sum_{i=0}^{2} \log _{2}\left|\mathcal{W}_{i}\right|
$$

a) $s \leq N_{E}^{\prime} \leq \min \left\{r_{1}, r_{2}\right\}$ : In this case, for $t=1,2$,

$$
\begin{aligned}
& \log _{2}\left|\mathcal{W}_{0}\right|+\log _{2}\left|\left\{i_{3}\right\}\right|=n\left(N_{E}^{\prime} R-\delta_{n}\right) \\
& \log _{2}\left|\mathcal{W}_{t}\right|=\log _{2}\left|\left\{j_{t}\right\}\right|=n\left(\left(r_{t}-N_{E}^{\prime}\right) R-\delta_{n}\right) \\
& \lim _{n \rightarrow \infty} \frac{1}{n} d\left(H\left(\tilde{\mathbf{X}}^{n}\right)\right)=\sum_{t=1}^{2}\left(r_{t}-N_{E}^{\prime}\right)+N_{E}^{\prime}
\end{aligned}
$$

Applying (28), (23) and (24) to (22), we find $\lim _{n \rightarrow \infty} \frac{1}{n} d\left(H\left(W_{0}, W_{1}, W_{2} \mid \tilde{\mathbf{Y}}_{\gamma}^{n}\right)\right) \quad$ is lower bounded by $\sum_{t=1}^{2}\left(r_{t}-N_{E}^{\prime}\right)+d_{0}$, which equals $\lim _{n \rightarrow \infty} \frac{1}{n} d\left(H\left(W_{0}, W_{1}, W_{2}\right)\right)$.

b) If $0 \leq N_{E}^{\prime}<s$, it can be verified that $\lim _{n \rightarrow \infty} \frac{1}{n} d\left(H\left(\tilde{\mathbf{X}}^{n}\right)\right)=r_{0}$. Therefore

$$
\lim _{n \rightarrow \infty} \frac{1}{n} d\left(H\left(W_{0}, W_{1}, W_{2} \mid \tilde{\mathbf{Y}}_{\gamma}^{n}\right)\right) \geq r_{0}-N_{E}
$$

which equals $\lim _{n \rightarrow \infty} \frac{1}{n} d\left(H\left(W_{0}, W_{1}, W_{2}\right)\right)$.

Hence we have proved (10) for both cases.

\section{F. Strong Secrecy for Arbitrarily Varying Channel}

In this section, we briefly outline the necessary changes in order to prove the strong secrecy requirement (4) when the eavesdropper channel is arbitrarily varying:

a) As shown in [12], to ensure secrecy when the eavesdropper channel is arbitrarily varying, "correlation elimination" [16] should be used. A coding scheme implied by this technique uses $K$ codebooks instead of one codebook. Each time the transmitter randomly chooses one codebook to use and reveals its choice as a public message.

b) As we have seen in Lemma 1, the rate of each bin is $N_{E} R$, which is smaller than the rate that the eavesdropper can decode, which is $R_{e}=N_{E} C\left(P / r_{0}\right)$. To ensure secrecy, we must amplify the bin size. This is done by using $K_{s}=2^{n\left(R_{e}-N_{E} R+2 \delta_{n}\right)}$ codebooks. Each time the transmitter chooses one codebook to use and transmits its choice to the two intended receivers as a common confidential message.

The coding scheme combines the two solutions above: The transmitter uses a collection of codebooks $\mathcal{C}^{1}, \ldots, \mathcal{C}^{K}$, each $\mathcal{C}^{k}$ composed of a collection of sub-codebooks denoted by $\mathcal{C}_{t, v}^{k}$, $1 \leq t \leq 3$ and $0 \leq v \leq K_{s}-1$. Each $\mathcal{C}_{t, v}^{k}$ is generated and labeled as shown in Section V-A and Section V-B.

a) Let $K^{\prime}$ and $K^{\prime \prime}$ be random variables uniformly distributed over $\{1, \ldots, K\}$ and $\left\{1, \ldots, K_{s}\right\}$ respectively. The transmitter chooses the sub-codebook $\mathcal{C}_{t, K^{\prime \prime}}^{K^{\prime}}, t=1,2,3$ and encode the confidential messages as in Section V-C. b) The transmitter transmits $K^{\prime}$ as a public message, and $K^{\prime \prime}$ as a common confidential message to both receivers.

The receivers first decode $K^{\prime}$ and $K^{\prime \prime}$ and use the subcodebook $\mathcal{C}_{t, K^{\prime \prime}}^{K^{\prime}}, t=1,2,3$ in $\mathcal{C}^{K^{\prime}}$ to decode the confidential messages as shown in Section V-D. It can be shown that the communication overhead for transmitting $K^{\prime}$ and $K^{\prime \prime}$ does not reduce the achieved secrecy degrees of freedom and Theorem 1 still holds.

\section{CONCLUSION}

In this work, we have introduced a new type of binning scheme. Through this binning scheme, we characterized the secrecy degrees of freedom region for a two-receiver MIMO broadcast wiretap channel where the eavesdropper channel is memoryless and arbitrarily varying for any given number of antennas.

\section{REFERENCES}

[1] C. E. Shannon, "Communication Theory of Secrecy Systems," Bell System Technical Journal, vol. 28, no. 4, pp. 656-715, September 1949.

[2] I. Csiszár and J. Körner, "Broadcast Channels with Confidential Messages," IEEE Transactions on Information Theory, vol. 24, no. 3, pp. 339-348, May 1978.

[3] E. Tekin and A. Yener, "The General Gaussian Multiple Access and Two-Way Wire-Tap Channels: Achievable Rates and Cooperative Jamming," IEEE Transactions on Information Theory, vol. 54, no. 6, pp. 2735-2751, June 2008.

[4] Y. Liang, G. Kramer, H. V. Poor, and S. Shamai, "Compound Wiretap Channels," Eurasip Journal on Wireless Communication and Networking, Special issue in Wireless Physical Layer Security, vol. 2009, Article ID 142374, 12 pages, 2009, doi:10.1155/2009/142374.

[5] L. Lai and H. El Gamal, "Cooperation for Secrecy: The RelayEavesdropper Channel," IEEE Transactions on Information Theory, vol. 54, no. 9, pp. 4005-4019, September 2008.

[6] E. Ekrem and S. Ulukus, "The Secrecy Capacity Region of the Gaussian MIMO Multi-receiver Wiretap Channel," IEEE Transactions on Information Theory, vol. 57, no. 4, pp. 2083-2114, April 2011.

[7] A. Khisti and G. Wornell, "Secure Transmission with Multiple AntennasI: The MISOME Wiretap Channel," IEEE Transactions on Information Theory, vol. 56, no. 7, pp. 3088-3104, July 2010.

[8] — , "Secure Transmission with Multiple Antennas-II: The MIMOME Wiretap Channel," IEEE Transactions on Information Theory, vol. 56, no. 11, pp. 5515-5532, November 2010.

[9] R. Liu, T. Liu, and H. V. Poor, "Multiple-input Multiple-output Gaussian Broadcast Channels with Confidential Messages," IEEE Transactions on Information Theory, vol. 56, no. 9, pp. 4215-4227, September 2010.

[10] M. Kobayashi, Y. Liang, S. Shamai, and M. Debbah, "On the Compound MIMO Broadcast Channels with Confidential Messages," in IEEE International Symposium on Information Theory, June 2009.

[11] E. MolavianJazi, "Secure Communication Over Arbitrarily Varying Wiretap Channels," Master Thesis, December 2009, available online at http://etd.nd.edu/ETD-db/theses/available/etd-12112009112419/unrestricted/MolavianJaziE122009.pdf.

[12] X. He and A. Yener, "MIMO Wiretap Channels with Arbitrarily Varying Eavesdropper Channel States," submitted to the IEEE Transactions on Information Theory, July, 2010, available online at http://arxiv.org/abs/1007.4801.

[13] A. Khisti, D. Silva, and F. Kschischang, "Secure Broadcast Codes over linear determintic channels," in IEEE International Symposium on Information Theory, May 2010.

[14] E. Ekrem and S. Ulukus, "Degrees of Freedom Region of the Gaussian MIMO Broadcast Channel with Common and Private Messages," in IEEE Global Telecommunication Conference, December 2010.

[15] S. Goel and R. Negi, "Guaranteeing Secrecy using Artificial Noise," IEEE Transactions on Wireless Communications, vol. 7, no. 6, pp. 21802189, June 2008.

[16] R. Ahlswede, "Elimination of correlation in random codes for arbitrarily varying channels," Probability Theory and Related Fields, vol. 44, no. 2, pp. 159-175, 1978. 